\title{
Prevalence of modifiable cardiovascular risk factors in long-term renal transplant patients
}

\author{
Vadamalai Vivek' \\ Sunil Bhandari ${ }^{1,2}$ \\ 'Department of Renal Medicine, \\ Hull and East Yorkshire Hospitals \\ NHS Trust and Hull York Medical \\ School, East Yorkshire, UK; \\ ${ }^{2}$ Statewide Renal Services, \\ Department of Renal Medicine, \\ Royal Prince Alfred Hospital, \\ Camperdown, Sydney, Australia
}

Correspondence: Sunil Bhandari Department of Renal Medicine, Hull Royal Infirmary, Hull and East Yorkshire Hospitals NHS Trust and Hull York Medical School, East Yorkshire HU3 2JZ, UK

Tel +44 I482 674556

Fax +44 I482 674998

Email sunil.Bhandari@hey.nhs.uk
This article was published in the following Dove Press journal: International Journal of Nephrology and Renovascular Disease 9 December 2010

Number of times this article has been viewed

Background: Cardiovascular disease accounts for the majority of morbidity and mortality in renal transplant patients. This relates to a number of modifiable and nonmodifiable risk factors, including new onset diabetes after transplantation (NODAT). We examined the prevalence of these risk factors in a cohort of 126 renal transplant patients.

Methods: A retrospective cross-sectional study of 94 nondiabetic post-transplant (ND) patients (mean age $45.7 \pm 13.5$ years) and 32 NODAT patients ( $55.2 \pm 9.6$ years) was performed. Univariate linear regression analysis was used to identify potential factors that affected cardiovascular events. Multivariable analysis was performed on those factors found to achieve a $P$ value of less than 0.20 after univariate analysis to test for significance in relation to cardiovascular risk as the primary factor.

Results: Mean serum creatinine levels were $131.1 \pm 4.3 \mu \mathrm{mol} / \mathrm{L}$ and $135.2 \pm 4.9 \mu \mathrm{mol} / \mathrm{L}$ at $96.9 \pm 8.7$ and $79.4 \pm 14.1$ months post-transplantation, respectively. Systolic pressure and pulse pressure were significantly higher in NODAT patients $(P=0.016$ and $P<0.005)$. Adequate target blood pressures were obtained in $80 \%$ of patients. Low-density lipoprotein and high-density lipoprotein cholesterol were reduced in $\operatorname{NODAT}(P=0.04$ and $P=0.005)$. Homocysteine was similarly elevated in both groups ( 17.5 and $15.6 \mu \mathrm{mol} / \mathrm{L}$, respectively). Coronary events and/ or coronary disease were present in $19.1 \%$ of ND and $37.5 \%$ of NODAT patients $(P<0.05)$. Cardiac deaths were three-fold more common ( $25 \%$ versus $7.4 \%$ ) in patients with NODAT. Univariate analysis revealed diabetes and age, and subsequent multivariable analysis revealed age only, as being significantly associated with cardiovascular outcomes.

Conclusions: Cardiac events are more common in patients with NODAT. Age is an important determinant of cardiovascular risk.

Keywords: blood pressure, cardiovascular risk, diabetes, post-transplant, renal, mortality

\section{Introduction}

Compared with the general population, cardiovascular disease in renal transplant recipients accounts for the majority of their morbidity and mortality. ${ }^{1-3}$ Although cardiovascular mortality has improved in transplant recipients compared with dialysis patients, it remains a significant problem. ${ }^{4}$ The estimated incidence of ischemic heart disease in renal transplant recipients is approximately $15 \%$ in comparison with $4 \%$ in the general population. ${ }^{5}$ This relates to a number of traditional potentially modifiable risk factors, including lipid abnormalities, diabetes mellitus, hypertension, tobacco intake, and elevated pulse pressure, and nonmodifiable risk factors, including gender, age, and family history. Newer potential risk factors identified include fibrinogen levels and elevated homocysteine levels. ${ }^{6}$ Other potential risk factors related specifically to 
transplantation include immunosuppressive therapy, acute rejection episodes, cytomegalovirus infection, anemia, proteinuria, and new onset diabetes after transplantation (NODAT). More recently emerging evidence has suggested that fibroblast growth factor-23, vitamin D, and disorders of mineral metabolism (including hyperparathyroidism, and calcium and phosphate levels) in relation to vascular calcification and arterial stiffness may be important in cardiovascular disease progression and cardiac events. Mechanistic factors involving inflammation, oxidative stress, and endothelial injury may all interact in this complex cardiovascular milieu to create an adverse risk in this population. ${ }^{7-9}$

Approximately $20 \%$ of recent transplant recipients are diabetic at the time of transplantation. Post-transplant diabetes is relatively common and is on the increase, occurring in up to $2 \%-46 \%$ of patients, especially with the use of newer immunosuppressive therapies. ${ }^{10}$ It represents an additional major risk factor for subsequent cardiac disease. Indeed, fasting blood glucose values in the upper normal range are associated with an increased risk for death from cardiovascular causes. ${ }^{11}$ Impaired nonoxidative glucose disposal explains the insulin resistance observed in NODAT similar to that observed in patients with type 2 diabetes mellitus in the general population. ${ }^{12}$ However, the extent of the problem in patients with long-standing transplants remains obscure.

Experience from our review of diabetic patients receiving a renal allograft indicates a high mortality from cardiac and infectious causes (unpublished data, Bhandari et al). At this center, historically almost $48 \%$ of diabetics diagnosed pretransplantation have died within five years of transplantation from cardiac causes, while a further $29 \%$ have died from infective causes. The aim of the present study was to examine cardiac risk factors (lipids, homocysteine, smoking, glycemia, duration of transplantation, serum albumin, blood pressure, uric acid, pulse pressure, age and gender, immunosuppressive regime, use of statins, use of angiotensin-converting enzyme inhibitors, and etiology of primary renal disease) in a cohort of Australian renal transplant recipients with and without NODAT and to compare the incidence of cardiac events after transplantation.

\section{Patient and methods}

We retrospectively performed a cross-sectional analysis using the departmental renal data system in a single regional renal transplant center of patients receiving renal allografts (deceased donor, live related/unrelated) from 1978 to 2000. All patients were aged less than 60 years at the time of transplantation. Patients with diabetes prior to renal allograft transplantation were excluded from the study. Patients with a documented or known prior cardiovascular event were not included in the study. Patients were classified into two groups, ie, nondiabetics (ND) and those with NODAT. Diabetes prior to transplantation was defined according to standard criteria for diagnosis, and any patient with a known history of documented diabetes at any time (continuous or for a short period), whether requiring dietary glucose restriction or oral hypoglycemic agents or insulin, was excluded from the study population. NODAT was defined as no pretransplant diabetes and development of diabetes post-transplantation. A fasting glucose of $>6 \mathrm{mmol} / \mathrm{L}$, a random glucose of $>11.1 \mathrm{mmol} / \mathrm{L}$, or the use of diet or oral hypoglycemic agents and/or insulin to normalize blood glucose was considered diagnostic for diabetes. ND patients had neither any pretransplant diabetes nor any new onset diabetes mellitus after transplantation. A fasting/random glucose of $<5.5 \mathrm{mmol} / \mathrm{L}$ was considered to be ND. Patients were not screened for impaired glucose tolerance. We investigated a total of 126 patients, comprising 94 ND (56 male and 38 female) and 32 NODAT patients (16 male and 16 female). Cardiovascular events were identified from case records. Criteria for diagnosis of a cardiovascular event were acute myocardial infarction, hospitalization for acute coronary syndrome, hospitalization for symptomatic angina, percutaneous coronary intervention, or coronary bypass surgery (after a diagnosis of NODAT or in the ND group). Stress testing and requirement for cardiac angiography was considered at the discretion of the caring physician in conjunction with a cardiology opinion. No predefined protocol was in place at the time of the study for cardiovascular surveillance. A history of hypertension and smoking was noted. A history or evidence of carotid artery disease or peripheral vascular disease was recorded for analysis. Three blood pressure recordings from separate clinic visits were recorded and averaged. Hypertension in this study was defined as a systolic pressure $>140 \mathrm{mmHg}$ and/or a diastolic pressure $>90 \mathrm{mmHg}$ according to the criteria of the Seventh Report of the Joint National Committee on Prevention, Detection, and evaluation of High Blood Pressure. Left ventricular hypertrophy was diagnosed in accordance with Sokolow-Lyon criteria.

Fasting plasma homocysteine levels were determined by high-performance liquid chromatography with fluorescence detection. Postload homocysteine was not performed because this has been shown to provide little more information in addition to measurement of homocysteine alone. ${ }^{13}$ Additional blood samples were analysed for plasma glucose, glycosylated hemoglobin ( $\mathrm{HbA1c}$ ), total cholesterol, high-density 
lipoprotein (HDL) cholesterol, triglycerides, uric acid, and serum albumin. Low-density lipoprotein (LDL) cholesterol was estimated with the Friedewald equation. HbAlc was determined in whole blood (ethylenediamine tetra-acetic acid tube). Triglycerides were measured by standard enzymatic calorimetric test.

\section{Statistical analysis}

All statistical analysis was carried out using SPSS for Windows (version 14; SPSS Inc., Chicago, IL). All continuous variables were expressed as means and standard deviation. Skewed data were logarithmically transformed to meet the assumptions of the statistical analysis. The
Mann-Whitney test was used for skewed distribution data. Two-sided unpaired $t$-tests, or one way analysis of variance, and Tukey's test were used to compare means between the two groups (ND and NODAT, see Table 1) to seek differences. Linear regression analysis was performed to evaluate univariate and multivariable clinical and biochemical predictors of cardiovascular events in relation to conventional cardiovascular risk factors. The dependent variable for which each potential risk factor (characteristic) was examined was cardiovascular events. Multivariable analysis was performed on those "putative" cardiovascular risk factors found to reach a $P$ value of less than 0.20 after univariate analysis to test for significance in relation to cardiovascular risk as

Table I Summary of analysis of patients

\begin{tabular}{|c|c|c|c|c|}
\hline Characteristic & $\begin{array}{l}\text { NODAT } \\
(\text { mean } \pm \text { SD) }\end{array}$ & $\begin{array}{l}\text { ND } \\
(\text { mean } \pm \text { SD) }\end{array}$ & $\begin{array}{l}P \text { values initial } \\
\text { analysis }\end{array}$ & $\begin{array}{l}P \text { values } \\
\text { multivariable } \\
\text { analysis }\end{array}$ \\
\hline \multicolumn{5}{|l|}{ Patient characteristics } \\
\hline Age & $55.2 \pm 9.6$ & $45.69 \pm 13.54$ & 0.001 & 0.001 \\
\hline $\operatorname{Sex}(M: F)$ & $16: 16$ & $56: 38$ & 0.3 & 0.685 \\
\hline Systolic BP (mmHg) & $134.22 \pm 15.7$ & $126.32 \pm 14.6$ & 0.016 & 0.308 \\
\hline Diastolic BP (mmHg) & $82.65 \pm 8.9$ & $81.48 \pm 8.48$ & 0.47 & 0.529 \\
\hline Pulse pressure & $51.56 \pm 12.98$ & $44.89 \pm 10.90$ & $<0.005$ & 0.12 \\
\hline Left ventricular hypertrophy & $7(23 \%)$ & $22(22 \%)$ & NS & \\
\hline Carotid artery disease & 0 & 7 (8\%) & NS & \\
\hline Peripheral vascular disease & $2(7.8 \%)$ & $5(5.3 \%)$ & NS & \\
\hline \multicolumn{5}{|l|}{ Smoking } \\
\hline Current & $4(12.5 \%)$ & $16(17 \%)$ & NS & \\
\hline Ex & $4(12.5 \%)$ & $8(8.5 \%)$ & NS & \\
\hline Nonsmoker & 17 (53.1\%) & $56(59.6 \%)$ & NS & \\
\hline Unknown & 7 (2I.9\%) & | 4 (|4.9\%) & NS & \\
\hline \multicolumn{5}{|l|}{ Biochemistry } \\
\hline HDL cholesterol (mmol/L) & $1.32 \pm 0.42$ & $1.62 \pm 0.53$ & 0.0048 & 0.660 \\
\hline LDL cholesterol (mmol/L) & $2.30 \pm 0.74$ & $2.67 \pm 0.77$ & 0.039 & 0.535 \\
\hline HDL/LDL cholesterol & $0.68 \pm 0.39$ & $0.68 \pm 0.34$ & 0.99 & 0.55 \\
\hline Total cholesterol (mmol/L) & $5.1 \pm 1.03$ & $4.73 \pm 0.94$ & 0.074 & 0.565 \\
\hline Triglycerides (mmol/L) & $1.76 \pm 0.82$ & $2.94 \pm 3.7$ & $<0.001$ & 0.454 \\
\hline Homocysteine ( $\mu \mathrm{mol} / \mathrm{L})$ & $15.57 \pm 4.26$ & $17.53 \pm 9.09$ & 0.26 & 0.524 \\
\hline Albumin (g/L) & $39.3 \pm 2.9$ & $38.96 \pm 2.84$ & 0.14 & 0.285 \\
\hline HbAlc (\%) & $7.08 \pm 1.33$ & $5.56 \pm 0.49$ & $<0.001$ & 0.313 \\
\hline Uric acid (mmol/L) & $\begin{array}{l}0.50 \pm 0.11 \\
(n)(\%)\end{array}$ & $\begin{array}{l}0.47 \pm 0.13 \\
(n)(\%)\end{array}$ & 0.28 & 0.523 \\
\hline $\begin{array}{l}\text { Immunosuppression } \\
\text { and number of agents }\end{array}$ & \multicolumn{3}{|c|}{$\begin{array}{l}\text { No differences in type of immunosuppression with cardiac events as the } \\
\text { dependent factor }\end{array}$} & NS \\
\hline Statin use $(n)$ & $22(69)$ & $46(49)$ & & \\
\hline ACEI & I I (34) & 45 (48) & & \\
\hline $\begin{array}{l}\text { Duration after transplant } \\
\text { (months) }\end{array}$ & $79.4 \pm 14.1$ & $96.9 \pm 8.7$ & 0.001 & NS \\
\hline \multicolumn{5}{|c|}{$\begin{array}{l}\text { Notes: } P \text { values in first column relate to analysis of variance for patients with and without new-onset diabetes after transplantation. The second column relates to } \\
\text { multivariable analysis of significant variables on univariate analysis using linear regression with cardiovascular events as the dependent variable for which only diabetes and } \\
\text { age had initial } P \text { values less than } 0.05 \text {. }\end{array}$} \\
\hline \multicolumn{5}{|c|}{$\begin{array}{l}\text { Abbreviations: ACEl, angiotensin-converting enzyme inhibitors; BP, blood pressure; HbAlc, glycosylated hemoglobin; HDL, high-density lipoprotein; LDL, low-density } \\
\text { lipoprotein; ND, nondiabetic post-transplantation; NODAT, new onset diabetes post-transplantation; M, male; F, female. }\end{array}$} \\
\hline
\end{tabular}


the dependent factor. This was carried out by a stepwise regression by sequentially removing variables one at a time until only significant variables remained. A value of $P<0.05$ was considered statistically significant.

\section{Results}

Ninety-four ND patients (56 men and 38 women) of mean age $45.7 \pm 13.5$ years and 32 NODAT patients (16 men and 16 women) of mean age $55.2 \pm 9.6$ years were studied (11 insulin-requiring and 21 requiring only an oral hypoglycemic agent). Causes of renal failure included autosomal dominant polycystic kidney disease $(n=14)$, reflux nephropathy $(n=13)$, Alport's kidney disease $(n=1)$, mesangiocapillary glomerulonephritis $(n=8)$, rapidly progressive glomerulonephritis $(n=6)$, chronic glomerulonephritis (11), undetermined etiology $(\mathrm{n}=14)$, analgesic nephropathy $(\mathrm{n}=8)$, IgA nephropathy $(\mathrm{n}=22)$, focal segmental glomerulosclerosis $(n=8)$, hypertension $(n=4)$, systemic lupus erythematosus $(n=3)$, obstructive uropathy $(n=3)$, dysplastic kidneys $(n=3)$, membranous glomerulonephritis $(n=3)$, hemolytic uremic syndrome $(n=1)$, renovascular disease $(n=1)$, postinfectious glomerulonephritis $(n=1)$, vasculitis $(\mathrm{n}=1)$ and cystinosis $(\mathrm{n}=1)$. Immunosuppressive regimes used in these patients were as detailed in Table 2.

Table 2 Summary of immunosuppression used in transplant patients and etiology of primary renal disease

\begin{tabular}{lll}
\hline Immunosuppression & $\mathrm{n}(\%)$ & $\mathrm{n}(\%)$ \\
Corticosteroids & $30(94)$ & $90(96)$ \\
Cyclosporine & $19(59)$ & $5 \mathrm{I}(54)$ \\
Tacrolimus & $3(9.3)$ & $6(6.3)$ \\
Azathioprine & $\mathrm{I} 7(53)$ & $54(57.4)$ \\
Mycophenolate & $9(28)$ & $23(24.5)$ \\
Sirolimus & $4(12.5)$ & $7(7.4)$ \\
Agents (n) & & \\
O & $0(0)$ & $1(1)$ \\
I & $1(3.1)$ & $6(6.4)$ \\
2 & $12(37.5)$ & $35(37.2)$ \\
3 & $19(59.4)$ & $52(55.3)$ \\
Cause of end-stage renal failure & & \\
IgA & $6(20)$ & $16(17.8)$ \\
Mesangio capillary glomerulonephritis & $2(6.7)$ & $6(6.7)$ \\
Polycystic kidney disease & $5(16.7)$ & $9(10)$ \\
Glomerulonephritis/chronic & $5(16.7)$ & $6(6.6)$ \\
Analgesics & $4(13.3)$ & $4(4.4)$ \\
Reflux/obstruction & $4(13.3)$ & $12(13.3)$ \\
Hypertension/nephrosclerosis & $3(10)$ & $1(1.1)$ \\
Vasculitis/systemic lupus erythematosus & $2(6.7)$ & $8(8.9)$ \\
Focal segmental glomerulosclerosis & $1(3.3)$ & $7(7.7)$ \\
Others & & $8(8.9)$ \\
Unknown & $14(15.6)$ \\
Dysplastic & $3(3.3)$ \\
\hline & &
\end{tabular}

NODAT patients were significantly older $(P=0.001)$, but there was no difference in gender and history of smoking. NODAT was more common in patients with polycystic kidney disease (16.7\% versus $10 \%)$ and in patients with analgesic nephropathy (13.3\% versus $4.4 \%$ ) as a cause of renal disease (Table 2). Most patients received double or triple immunosuppressive therapy (97\% and 93\% for NODAT and ND, respectively). Both glucose and HBA1c concentrations were significantly elevated in NODAT $(P<0.001$, Figure 1$)$ but the levels in this group were adequately controlled.

Univariate analysis revealed that both age $(P<0.001)$ and diabetes $(P<0.05)$ were significant risk factors for cardiovascular events as the dependent variable. However, after multivariable analysis, only age remained as the predominant significant risk factor $(P<0.001)$, while diabetes was no longer significant $(P=0.47)$.

\section{Renal function}

Mean serum creatinine concentrations were $131.1 \pm 4.3 \mu \mathrm{mol} / \mathrm{L}$ and $135.2 \pm 4.9 \mu \mathrm{mol} / \mathrm{L}(P=\mathrm{NS})$, respectively, between ND and NODAT patients at mean times of $96.9 \pm 8.7$ and $79.4 \pm 14.1$ months post-transplantation.

\section{Lipids}

HMG-CoA reductase inhibitor (statin) therapy was used in $54 \%$ of patients. LDL cholesterol levels were lower in the NODAT group $(2.3 \pm 0.74 \mathrm{mmol} / \mathrm{L}$ versus $2.7 \pm 0.77 \mathrm{mmol} / \mathrm{L}$, respectively, $P=0.039$ ). Forty-six percent of patients had total cholesterol levels $>5 \mathrm{mmol} / \mathrm{L}$ (12 NODAT and $46 \mathrm{ND}$ ), and $34 \%$ had low-density lipoprotein (LDL) cholesterol levels greater than $3 \mathrm{mmol} / \mathrm{L}$ (35 ND and 8 NODAT) despite lipidlowering therapy. High-density lipoprotein (HDL) cholesterol

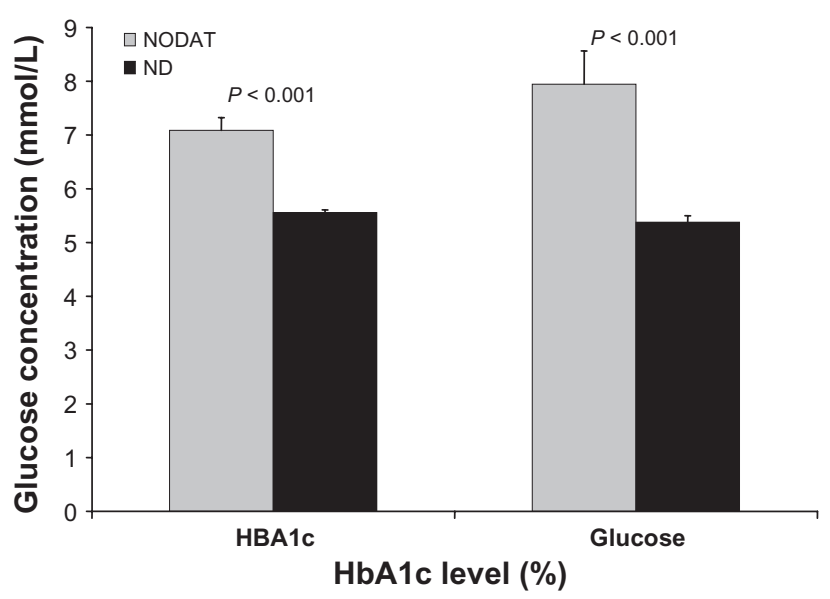

Figure I Glycemic control as measured by HBAIc levels and fasting glucose in transplant patients with (NODAT) and without (ND) new onset diabetes after transplantation. 
concentrations were lower in NODAT patients. The median total cholesterol:HDL ratio was 3.3 (range 1.53-6.8), with $11 \%$ of patients having ratios $>5$. Triglyceride levels were markedly elevated in patients with NODAT (Figure 2).

\section{Homocysteine}

Homocysteine levels were similarly elevated in both groups (17.5 $\mu \mathrm{mol} / \mathrm{L}$ and $15.6 \mu \mathrm{mol} / \mathrm{L}$, respectively, Figure 3), and $78 \%$ had a level greater than $12 \mu \mathrm{mol} / \mathrm{L}$. There was a positive correlation of homocysteine with serum creatinine $(\mathrm{r}=0.3)$, age $(\mathrm{r}=0.32)$, and serum albumin $(\mathrm{r}=0.4)$. This correlation was more pronounced in patients with NODAT. Previously it has been noted that transplant patients on cyclosporine tend to have higher homocysteine levels. ${ }^{14}$ However, we found no such trend in this select cohort of transplant patients.

\section{Cardiovascular disease}

A history of or current hypertension requiring therapy was present in $68 \%$ of ND and $87 \%$ of NODAT patients. Systolic blood pressure and pulse pressure were significantly higher in NODAT $(P=0.016$ and $P<0.005$, respectively, Figure 4). ACE inhibitors and/or angiotensin II receptor antagonists were used in $40 \%$ of NODAT and $48 \%$ of ND patients. Eleven female and five male patients had a systolic blood pressure greater than $140 \mathrm{mmHg}$ (all on treatment), four female and six male patients had a diastolic blood pressure greater than $90 \mathrm{mmHg}$ (all but one on therapy), and three each had both elevated systolic and diastolic blood pressures. Adequate target blood pressures $(<140 / 90 \mathrm{mmHg})$ were obtained in $80 \%$ of patients. Twenty-nine patients $(23 \%$ versus $22 \%$ for ND and NODAT, respectively) had electrocardiographic criteria for left ventricular hypertrophy, with no statistically significant difference found between groups.

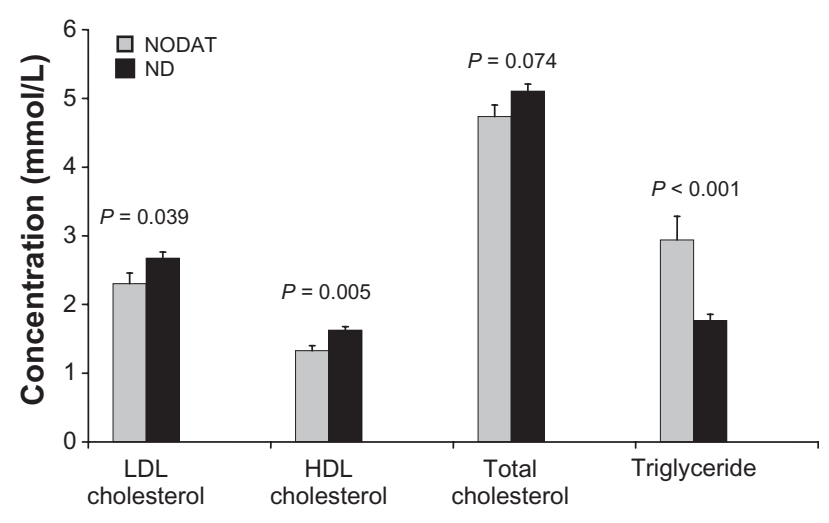

Figure 2 Lipid profiles in transplant patients with (NODAT) and without (ND) in the presence of new onset diabetes after transplantation.

Abbreviations: LDL, low-density lipoprotein; HDL, high-density lipoprotein; ND, nondiabetic; NODAT, new onset diabetes after transplantation.

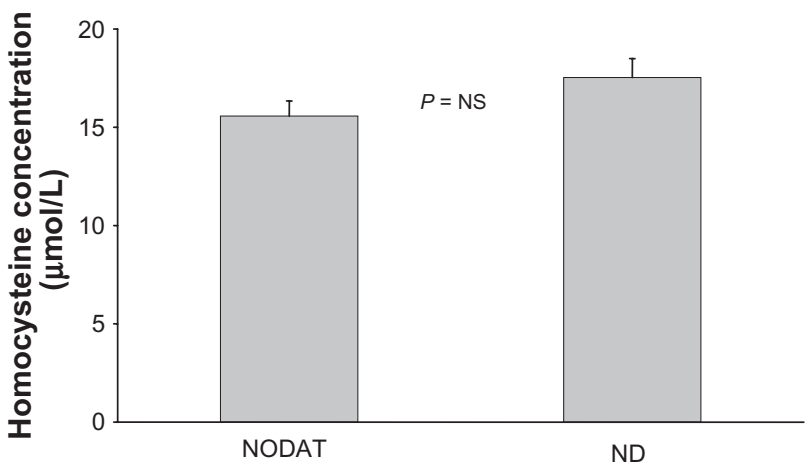

Figure 3 Mean fasting Homocystine levels in patents with (NODAT) and without (ND) new onset diabetes after transplantation.

Albumin and uric acid levels were similar in both groups and not related to cardiac events.

In total, seven patients $(8 \%)$ in the ND group had documented evidence of carotid artery disease versus none in the NODAT group, but the data set was incomplete, and analysis revealed no significance association with cardiac events. Seven patients also had evidence of peripheral vascular disease, again with an incomplete data set, and no association was found with cardiovascular events.

Symptomatic cardiovascular events were present in $19.1 \%(\mathrm{n}=18)$ of ND and $37.5 \%(\mathrm{n}=12)$ of NODAT patients $(P<0.05)$. For NODAT patients, there were six documented myocardial infarctions, for one of which surgery was subsequently required. Six patients had acute coronary syndrome or angina. Three patients with acute coronary syndrome or who had had a myocardial infarction required subsequent angiography and/or angioplasty. For ND patients, there were 14 myocardial infarctions, three of which had subsequent coronary artery bypass grafts. Four patients had acute coronary syndrome or angina. Seven patients in the ND group of 18 patients with cardiovascular events had

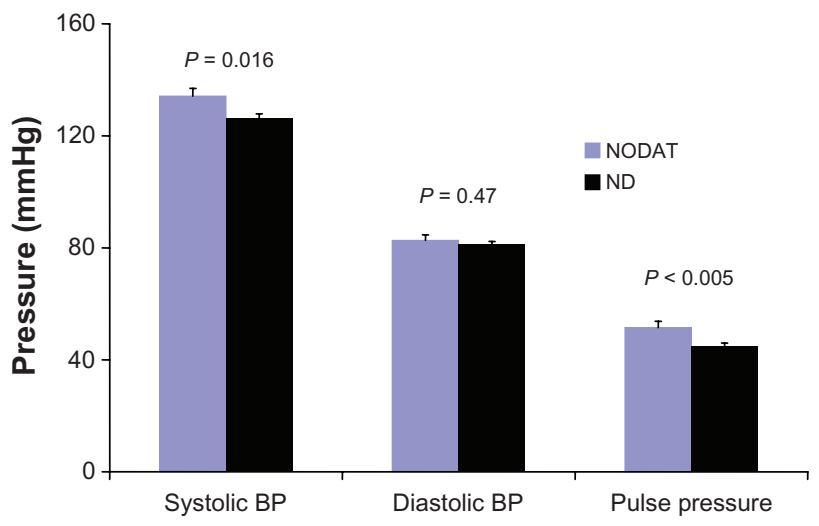

Figure 4 Blood pressure control in transplant patients with (NODAT) and without (ND) new onset diabetes after transplantation.

Abbreviations: BP, blood pressure; ND, nondiabetics; NODAT, new onset diabetes after transplantation. 
angiography and/or angioplasty. Using cardiovascular events as the dependent variable, analysis of variance factored age and diabetes as candidate variables and age as a potential adverse risk factor for cardiac event rates after multivariable analysis (Table 1). Over the entire follow-up period, there were 35 (27.8\%) deaths, of which 18 (56.3\%) occurred in the NODAT group and $16(17 \%)$ in the ND group. In the NODAT group there were eight (25\%) deaths reported as being cardiac in nature. The remaining deaths were due to sepsis $(n=7)$, unknown cause $(n=2)$, and carcinoma $(n=1)$. In the ND group, seven $(7.4 \%)$ deaths were reported as cardiac and nine noncardiac $(\operatorname{sepsis} n=3$, carcinoma $n=2$, unknown $n=3$, and stroke $n=1)$.

\section{Discussion}

Cardiovascular risk factors were highly prevalent in this cohort of transplant patients. More than $90 \%$ of patients had recognized cardiovascular risk factors. Despite reasonably good blood pressure and lipid control, the incidence of cardiovascular events overall in this cohort of patients was $23 \%$. Our objective was to determine whether the development of NODAT might represent a mediator in the relationship of traditional cardiovascular risk factors, metabolic abnormalities, and cardiovascular disease. Cardiovascular events were especially more prevalent in those patients with NODAT (37.5\% versus 19.1\%). Both age and male gender are important risk factors for cardiovascular disease in transplant patients. ${ }^{2}$ In considering what mechanisms may be responsible for the higher cardiovascular risk among individuals with NODAT, we observed that NODAT individuals were older than those with ND, but there was no gender difference. ND patients also had functioning grafts for a longer period of time (on average 16 months). However, although there was a strong association of NODAT with cardiac complications in the unadjusted analysis, the association was attenuated after statistical adjustment for demographic parameters and confounding risk factors previously associated with cardiovascular disease (Table 1). Mortality was significantly higher in the NODAT group (56.3\% versus $17 \%$ ) over the course of follow-up (mean 91 months), with cardiac deaths accounting for $12 \%$ of all deaths in this cohort of 126 transplant patients and occurring at least three-fold more often in patients with NODAT (25\% versus $7.4 \%)$.

Blood pressure was adequately controlled, but both systolic and pulse pressure were significantly higher in patients with NODAT. Good control of blood pressure perhaps is an essential part of management to preserve renal function and reduce overall cardiovascular risk.
Hypertension was prevalent but similar in both groups. In total, $75 \%-90 \%$ of patients with functioning renal transplants have hypertension and 55\% do not achieve target blood pressure. ${ }^{15,16}$ Our data indicate much better control, thus reducing cardiovascular risk and potentially improving long-term transplant outcomes.

Post-transplantation patients tend to have elevated total cholesterol, triglycerides, LDL, and normal or elevated HDL. ${ }^{10}$ Indeed, the prevalence of this pattern of biochemical changes occurs in up to $60 \%$ of patients, which may lead to increased future cardiovascular risk. This relates in part to immunosuppressive therapy, especially steroids, calcineurin inhibitors, and, more recently, rapamycin. ${ }^{17}$ Many randomized controlled trials in nontransplant patients have shown a reduction in cardiovascular risk via lowering of cholesterol concentrations. ${ }^{18,21}$ In transplant patients, currently only the ALERT (Assessment of Lescol in Renal Transplantation) study has shown a benefit of lipid-lowering therapy in this population. ${ }^{22,23}$ Although there was no reduction in primary endpoints, secondary endpoints showed a 35\% reduction of cardiac death and nonfatal myocardial infarction. This finding was significant and similar to other nontransplant studies. Statins may also have other additional pleiotropic effects beyond lowering LDL cholesterol, including nitric oxide production in endothelium, an independent antihypertensive effect, an antithrombotic effect through reduced thromboxane production, and an anti-inflammatory effect. ${ }^{19}$ Several reports indicate that use of statins early during transplantation correlates with improved renal allograft survival independent of cholesterol or triglyceride levels. ${ }^{24}$ LDL and HDL cholesterol levels were lower in NODAT patients, but the HDL:LDL ratio was higher overall in more patients with NODAT. HDL cholesterol is a strong independent predictor for the development of and death from ischemic heart disease. ${ }^{2,3}$ In addition, raised triglycerides increase the risk of ischemic heart disease, but this measure remains difficult to evaluate in the clinical context. ${ }^{2}$ Our data suggest that although lipid levels were reasonable, the lower LDL and HDL levels in the NODAT group could also reflect a higher prevalence of metabolic syndrome (reflected by NODAT). Therefore, in addition to more aggressive management of dyslipidemia, diet and exercise may be beneficial for optimal cardiovascular risk reduction. Indeed, examination of insulin resistance and factors such as body mass index and waist circumference may have been useful adjuncts in global assessment of this cohort of patients.

Homocysteine is a sulfur-containing amino acid, the metabolism of which depends on vitamin B12 and folic acid. 
Plasma levels of homocysteine are raised markedly in dialysis patients. Homocysteine levels continue to rise following renal or cardiac transplantation, independent of renal function. ${ }^{25,26}$ This has been associated with adverse cardiovascular morbidity and overall mortality. ${ }^{1}$ Elevated homocysteine levels and lipids are graded as independent risk factors for cardiovascular and generalized atherosclerotic disease comparable with smoking. ${ }^{13}$ A $5 \mu \mathrm{mol} / \mathrm{L}$ increment in fasting/nonfasting homocysteine levels is associated with a $25 \%-30 \%$ increase in coronary heart disease risk. ${ }^{27}$ In this study, homocysteine levels were high in the majority of patients (83\%) and reduced with time post-transplantation, but remained significantly higher (approximately $5 \mu \mathrm{mol} / \mathrm{L}$ ) than the normal range. Serum folate levels were normal in this cohort of patients. Hyperhomocysteinemia perhaps contributes to the overall increased cardiovascular risk in this transplant group, but is not influenced by the presence of diabetes.

NODAT is a complication of immunosuppression. Its incidence amounts to approximately $18 \%{ }^{28}$ Many risk factors have been proposed for the development for NODAT, including a family history of diabetes mellitus, human leukocyte antigens (HLA) A28, A30, B8, B27, and BW42, AfricanAmerican and Hispanic ethnicity, tacrolimus use, and steroid dose. ${ }^{10,28,29}$ Risk factors with limited supporting evidence include pretransplant renal failure due to interstitial nephritis and polycystic kidney disease, deceased donor rather than haploidentical living graft, ${ }^{30}$ number of episodes of acute rejection, longer duration of dialysis prior to transplantation, interleukin-6 gene promoter polymorphism, hepatitis $\mathrm{C}$ virus infection, and higher body mass index. ${ }^{10}$

Steroids and calcineurin antagonists contribute to the increased prevalence of NODAT. ${ }^{31}$ The need to avoid NODAT has to be a consideration in the choice of immunosuppressive regime. However, modification to the immunosuppression regime needs to be weighed carefully against the risk of rejection.

The impact of NODAT on cardiovascular morbidity and mortality is somewhat obscure. Mortality was significantly higher in our NODAT group, but age was the most significant risk factor, with the ND patients being a decade younger. Therefore, the effects of good glycemic control are less clear. Pretransplant diabetes mellitus is an established and powerful predictor for death from ischemic heart disease in renal transplant recipients. ${ }^{3}$ Diabetic patients have a high incidence of asymptomatic cardiovascular disease. ${ }^{20}$ Therefore, in this population, given the high incidence of NODAT (25.4\%), it might be pertinent to monitor these patients prospectively and perhaps offer aggressive risk factor management and elective angiography and/or angioplasty before significant vascular complications occur. Several authors have suggested performing an oral glucose tolerance test to detect impaired glucose tolerance and instituting cardiovascular risk reduction strategies even before a diagnosis of NODAT is made. ${ }^{32}$ Prospective comparative data are needed to examine this phenomenon more closely. Perhaps good glycemic control may have beneficially contributed to the relatively good survival in our cohort.

\section{Summary}

This study presents some intriguing findings in patients with long-term functioning renal transplants. Older age represents the strongest risk factor for cardiovascular events. Although NODAT is a significant risk factor in univariate analysis, its effect is diluted in multivariable analysis, perhaps by the large age difference. However, we believe that vigilance in detecting and tightly controlling or preventing NODAT may be important for limiting cardiovascular risk and eventual death with a functioning graft. Equally it may well be that diabetes per se does not lead to cardiac disease, but is a surrogate marker for it. Perhaps immunosuppressive therapy should be tailored to reducing this increasing problem and thereby avoiding cardiovascular events. Hence equally potent drugs are required without the adverse effects on the metabolic profile. The strong association between age and cardiovascular risk in transplant patients may have implications with regards to addressing cardiovascular risk more aggressively in older potential transplant recipients.

This study has several limitations. One acknowledges that this was a retrospective observational cross-sectional study in an Australian cohort of transplant recipients and that small numbers were used. However, it does highlight some interesting findings. Silent myocardial ischemia is common in this high-risk population, and we cannot exclude a number of patients having occult coronary artery disease with few clinical symptoms. In relation to metabolic syndrome, data on waist circumference and proteinuria may have been helpful but were not available. Therefore, prospective studies are necessary to investigate any potential causal relationship between NODAT and cardiovascular endpoints. Larger numbers would be necessary to remove possible type 2 errors occurring and to see if interventions to reduce risk factors lead to overt reductions in cardiovascular risk. No examination of other risk factors, including anemia, was undertaken, and therefore represents another limitation to the study. 


\section{Disclosure}

The authors report no conflict of interest in this work.

\section{References}

1. Moustapha A, Naso A, Nahlawi M, et al. Prospective study of hyperhomocysteinemia as an adverse cardiovascular risk factor in end-stage renal disease. Circulation. 1998;97:138-141.

2. Kasiske BL, Guijarro C, Massy ZA, Wiederkehr MR, Ma JZ. Cardiovascular disease after renal transplantation. J Am Soc Nephrol. 1996; 7:158-165.

3. Aakhus S, Dahl K, Wideroe TE. Cardiovascular morbidity and risk factors in renal transplant patients. Nephrol Dial Transplant. 1999;14: 648-654.

4. Arend SM, Mallat MJ, Westendorp RJ, van der Woude FJ, van Es LA. Patient survival after renal transplantation; more than 25 years follow-up. Nephrol Dial Transplant. 1997;12:1672-1679.

5. Kasiske BL. Risk factors for accelerated atherosclerosis in renal transplant recipients. Am J Med. 1988;84:985-992.

6. Ducloux D, Kazory A, Chalopin JM. Predicting coronary heart disease in renal transplant recipients: A prospective study. Kidney Int. 2004;66: $441-447$.

7. Wolf M, Shah A, Gutierrez O, et al. Vitamin D levels and early mortality among incident haemodialysis patients. Kidney Int. 2007;72: 1004-1013

8. Mirza MA, Larsson A, Melhus H, Lind L, Larsson TE. Serum intact FGF-23 associated with LV mass, hypertrophy and geometry in elderly population. Atherosclerosis. 2009;5:1010-1016.

9. de Silva R, Rigby AS, Witte KK, et al. Anemia, renal dysfunction and their interaction in patients with chronic heart failure. Am J Cardiol. 2006;98:391-398.

10. Kasiske BL, Snyder JJ, Gilbertson D, Matas AJ. Diabetes mellitus after kidney transplantation in the United States. Am J Transplant. 2003;3: 178-185.

11. Cosio FG, Kudva Y, van der Velde M, et al. New onset hyperglycemia and diabetes are associated with increased cardiovascular risk after kidney transplantation. Kidney Int. 2005;67:2415-2421.

12. Ekstrand AV, Eriksson JG, Gronhagen-Riska C, Ahonen PJ, Groop LC. Insulin resistance and insulin deficiency in the pathogenesis of post transplantation diabetes in man. Transplantation. 1992;53:563-569.

13. Vermeulen EG, Stehouwer CD, Twisk JW, et al. Effect of homocysteinelowering treatment with folic acid plus vitamin B6 on progression of subclinical atherosclerosis: A randomized, placebo-controlled trial. Lancet. 2000;355:517-522.

14. Arnadottir M, Hultberg B, Vladov V, Nilsson-Ehle P, Thysell H. Hyperhomocysteinemia in cyclosporine-treated renal transplant recipients. Transplantation. 1996;61:509-512.

15. Ojo AO. Cardiovascular complications after renal transplantation and their prevention. Transplantation. 2006;82:603-611.

16. Opelz G, Zeier M, Laux G, Morath C, Dohler B. No improvement of patient or graft survival in transplant recipients treated with angiotensinconverting enzyme inhibitors or angiotensin II type 1 receptor blockers: A collaborative transplant study report. J Am Soc Nephrol. 2006; 17:3257-3262.

17. Chueh SC, Kahan BD. Dyslipidemia in renal transplant recipients treated with a sirolimus and cyclosporine-based immunosuppressive regimen: Incidence, risk factors, progression, and prognosis. Transplantation. 2003;76:375-382.
18. Randomized trial of cholesterol lowering in 4444 patients with coronary heart disease: The Scandinavian Simvastatin Survival Study (4S). Lancet. 1994;344:1383-1389.

19. Sacks FM, Pfeffer MA, Moye LA, et al. The effect of pravastatin on coronary events after myocardial infarction in patients with average cholesterol levels. Cholesterol and Recurrent Events Trial investigators. N Engl J Med. 1996;335:1001-1009.

20. The Long-Term Intervention with Pravastatin in Ischaemic Disease (LIPID) Study Group. Prevention of cardiovascular events and death with pravastatin in patients with coronary heart disease and a broad range of initial cholesterol levels. $N$ Engl J Med. 1998;339:1349-1357.

21. Baigent C, Keech A, Kearney PM, et al. Efficacy and safety of cholesterol-lowering treatment: Prospective meta-analysis of data from 90,056 participants in 14 randomized trials of statins. Lancet. 2005; 366:1267-1278.

22. Holdaas H, Fellstrom B, Jardine AG, et al. Effect of fluvastatin on cardiac outcomes in renal transplant recipients: A multicentre, randomized, placebo-controlled trial. Lancet. 2003;361:2024-2031.

23. Holdaas H, Fellstrom B, Jardine AG. Clinical practice guidelines for managing dyslipidemias in kidney transplant patients: Lessons to be learnt from the assessment of Lescol in renal transplantation (ALERT) trial. Am J Transplant. 2005;5:1574-1575.

24. Cosio FG, Pesavento TE, Pelletier RP, et al. Patient survival after renal transplantation III: The effects of statins. Am J Kidney Dis. 2002; 40:638-643.

25. Gupta A, Moustapha A, Jacobsen DW, et al. High homocysteine, low folate, and low vitamin B6 concentrations: Prevalent risk factors for vascular disease in heart transplant recipients. Transplantation. 1998; 65:544-550.

26. Massy ZA, Chadefaux-Vekemans B, Chevalier A, et al. Hyperhomocysteinaemia: A significant risk factor for cardiovascular disease in renal transplant recipients. Nephrol Dial Transplant. 1994;9:1103-1108.

27. Boushey CJ, Beresford SA, Omenn GS, Motulsky AG. A quantitative assessment of plasma homocysteine as a risk factor for vascular disease. Probable benefits of increasing folic acid intake. JAMA. 1995; 274:1049-1057.

28. Hjelmesaeth J, Hartmann A, Kofstad J, et al. Glucose intolerance after renal transplantation depends upon prednisolone dose and recipient age. Transplantation. 1997;64:979-983.

29. von Kiparski A, Frei D, Uhlschmid G, Largiader F, Binswanger U. Post-transplant diabetes mellitus in renal allograft recipients: A matchedpair control study. Nephrol Dial Transplant. 1990;5:220-225.

30. Boudreaux JP, McHugh L, Canafax DM, et al. The impact of cyclosporine and combination immunosuppression on the incidence of post transplant diabetes in renal allograft recipients. Transplantation. 1987;44:376-381.

31. Webster AC, Woodroffe RC, Taylor RS, Chapman JR, Craig JC. Tacrolimus versus ciclosporin as primary immunosuppression for kidney transplant recipients: Meta-analysis and meta-regression of randomized trial data. $B M J .2005 ; 331: 810$.

32. Sharif A, Moore RH, Baboolal K. The use of oral glucose tolerance tests to risk stratify for new-onset diabetes after transplantation: An under diagnosed phenomenon. Transplantation. 2006;82:1667-1672.

International Journal of Nephrology and Renovascular Disease

\section{Publish your work in this journal}

The International Journal of Nephrology and Renovascular Disease is an international, peer-reviewed open-access journal focusing on the pathophysiology of the kidney and vascular supply. Epidemiology, screening, diagnosis, and treatment interventions are covered as well as basic science, biochemical and immunological studies. The journal welcomes original research, clinical studies, reviews \& evaluations, expert opinion and commentary, case reports and extended reports. The manuscript management system is completely online and includes a very quick and fair peerreview system, which is all easy to use. Visit http://www.dovepress.com/ testimonials.php to read real quotes from published authors. 